\title{
Coordinated Operation of Parallel-Connected Inverters for Active Islanding Detection Using High Frequency Signal Injection
}

\author{
Fernando Briz, David Reigosa, Cristian Blanco, Juan Manuel Guerrero \\ University of Oviedo. Dept. of Elect., Computer \& System Engineering, Gijón, 33204, Spain. \\ fernando@isa.uniovi.es, reigosa@isa.uniovi.es, blancocristian@uniovi.es, guerrero@isa.uniovi.es
}

\begin{abstract}
The high frequency impedance measured at the terminals of inverters connected in a microgrid by means of the injection of a small magnitude, high frequency voltage, has been shown to be a reliable metric to detect islanding. While the implementation of this method is simple when only an inverter injects the high frequency signal, this case is of limited applicability. On the other hand, several concerns arise when multiple inverters work in parallel, primarily due to risk interference among inverters. Islanding detection using high frequency signal injection in microgrids with multiple parallelconnected inverters is studied in this paper. A strategy for the coordinated operation of the inverters, without the need of communications or pre-established roles is proposed. Simulation and experimental results will be provided to demonstrate the viability of the concept. ${ }^{1}$

Index Terms- Active islanding detection, grid impedance measurement, high frequency signal injection, distributed generation.
\end{abstract}

\section{I.- Introduction}

Microgrids are becoming a key concept for the integration of Distributed generation (DG) based on renewable and nonrenewable energy resources (photovoltaic, fuel cells, biomass, wind turbines, etc.), as well as Distributed storage (DS) systems. A feature of microgrids using DGs and DSs is that sources and loads can be distributed over a relatively large area. Because of the distributed nature, active elements of the microgrids should be controlled using local measurements, i.e. without dependence on communications systems [1].

Islanding detection is of special importance for the microgrid concept $[2,3,14]$. Islanding is defined as the situation in which DGs continue generating power when the microgrid is not connected to the utility grid. The requirements for islanding detection are regulated by local, regional and national authorities, standardizing institutions as IEEE and IEC also publishing recommendations for interconnecting distributed resources with the electric power systems [4-13]. As examples of these, the IEEE 1547 standard [4], the IEEE 929 standard [7], the UL 1741 standard [8] and the IEC standard [9] require islanding detection in less than 2 seconds after the islanding condition occurs. The German standard, DIN VDE 0126 [10] establishes that islanding detection methods should detect variations of the grid impedance of 1 $\mathrm{Ohm}$ in 2 seconds, $0.5 \mathrm{Ohm}$ in 5 seconds being established by Austrian and Swiss standards $[12,13]$.

1 This work was supported in part by the Research, Technological Development and Innovation Programs of the Ministry of Science and Innovation under grant MICINN-10-CSD2009-00046 and of the Spanish Ministry of Science and Innovation-ERDF under grant MICINN-10ENE2010-14941.
Islanding detection methods can be classified into two groups: Remote and local (inverter resident) $[2,3,14]$.

Remote methods are based on communications between the grid and the DGs. They are highly flexible and with no risk of non-detection zone (NDZ). However, they are usually expensive due to the need of a communication infrastructure [14], also the risk of communication breakdown exists.

Local methods are based on data at the DGs site, and are of two types: Passive and active. Passive methods measure a system parameter (under/over voltage, under/over frequency, etc), and compare it with a preset threshold. They are normally cheap to implement and grid friendly, as no additional signal is injected; however they present a large non-detection zone NDZ [14,15]. Active methods produce some kind of disturbance at the DGs output, and measure the grid response [14-24]. These methods have a lower NDZ, but can negatively impact the power quality; also interference among power converters trying to detect islanding simultaneously can occur.

Three potential scenarios can be considered for the evaluation of islanding detection methods. [3]: 1) Single inverter, 2) Multi-inverter, when multiple parallel connected inverters interact at one PCC, and a major portion, or all the power produced by the DGs is exported through the PCC, and 3) Microgrid, in which local loads can consume a significant amount, or all the power produced by the DGs.

High frequency signal injection methods [17-22] are a type of active local methods, in which a high frequency signal is injected via inverter, islanding being detected from the measured response. The implementation of these methods is easy in case 1), when only a inverter injects the high frequency voltage, but is challenging in case 2), as interference among inverters can occur. However, the fact that the inverters operate at a frequency different from the grid frequency, makes them suitable for case 3), even in the event that the DGs output power exactly matches the local loads and not power flows through the PCC.

This paper analyzes islanding detection using high frequency signal injection in grids with multiple parallelconnected inverters. To prevent interference, all the inverters participating in the strategy inject a synchronized high frequency signal. In order to minimize the impact on the grid, the inverters can behave either as Voltage sources or as Current sources, having the ability to self-reconfigure their mode of operation, depending on the microgrid/grid condition, without the need of communications or preestablished master/slave roles. 


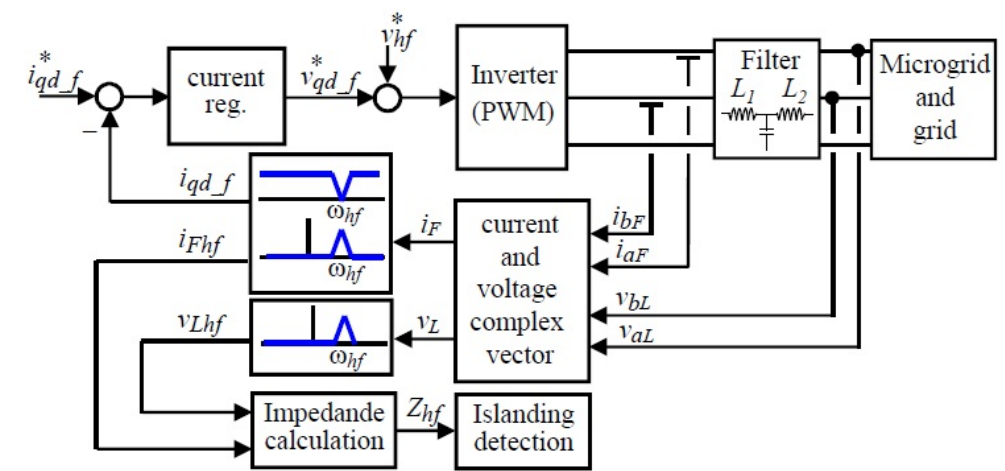

Fig. 1.- Block diagram of the master inverter.

\section{II.- Islanding detection using high frequency voltage injection}

Recently published works have shown that the high frequency impedance is a reliable metric to detect an islanding situation [17-22]. Voltage-source inverters (VSI) present in a microgrid (e.g. in controlled rectifiers used in APFs, back-to-back power converters, ...) can easily inject a low-magnitude, high-frequency voltage, from which the high frequency impedance is estimated. As an example, a rotating high frequency signal (1) can be injected, the induced high frequency current $i_{F h f}$ being (2), $Z_{h f}$ (3) being the high frequency impedance at the excitation frequency, with its phase angle being $\varphi_{Z}$, where $\omega_{h f}$ and $V_{h f}$ are the frequency and magnitude of the injected high frequency voltage.

$$
\begin{aligned}
& v_{h f}=V_{h f} e^{\mathrm{j} \omega h f t} \\
& i_{F h f}=\frac{V_{h f}}{Z_{h f}} e^{\mathrm{j}\left(\omega h f t-\varphi_{Z}\right)} \\
& Z_{h f}=R_{h f}+\mathrm{j} \omega_{h f} L_{h f} ; \varphi_{Z}=\operatorname{atan}\left(\frac{\omega_{h f} L_{h f}}{R_{h f}}\right)
\end{aligned}
$$

Fig. 1 shows the schematic implementation of the method. Fig. $2 \mathrm{a}$ and $2 \mathrm{~b}$ show the magnitude and phase of the high frequency impedance $Z_{h f}$, during a transition from island to grid, and from grid to island. The change in both metrics between islanding and grid condition are readily observable. Islanding detection is based on the rate of variation of $Z_{h f}$ with time, i.e. $\Delta Z_{h f} / \Delta t$. Comparison of $Z_{h f}$ with a threshold might also potentially be used [21]. Further discussion on other forms of high frequency excitation as well as on the implementation of this method can be found in $[21,22]$.

\section{III.- Islanding detection using high frequency voltage injection for the case of multiple inverters}

While simple for the case of a single inverter, the extension of the method described in Section II to the case of multiple inverters is not trivial, strategies that have been proposes to address this case are discussed in this section. In the discussion following, master inverters refers to inverters which inject a high frequency signal to detect the microgrid
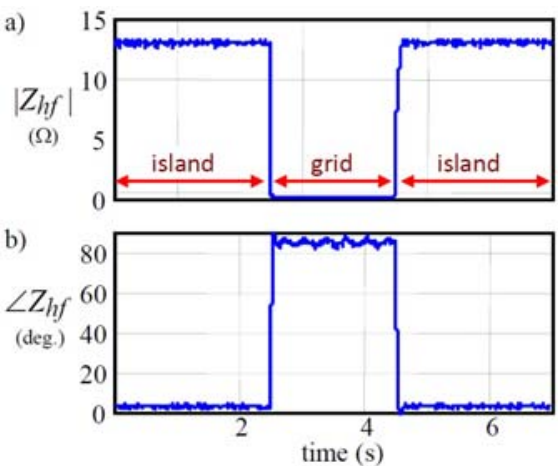

Fig. 2.- Experimentally measured high frequency impedance during island-grid transitions $V_{h f}=1.5 \mathrm{~V}, \omega_{h f}=-333 \mathrm{~Hz}$.

condition (grid/island), while slave inverters refers to inverters without such capability, but which still need to know the grid condition for their proper operation. This means that master inverters are responsible of informing the slave inverters of the grid condition. For the evaluation of the strategies, the following issues need to be taken into account:

- All the inverters in the microgrid participating in the strategy should know the microgrid condition (island/grid connected) within a time frame compatible with islanding detection standards.

- The adverse impact on the microgrid/grid power quality (THD), due to the injection of high frequency signals, should be kept as small as possible, and below the limits established by the connection standards [4-7].

- Use of separate communication channels is not allowed.

- The injection of an additional high frequency signal for communication purposes is acceptable, as this does not have the problems/concerns intrinsic to communications, like cost and the risk of communication breakdown. Details on the use of the secondary high frequency signal can be found in $[21,22]$.

a) Static, single master inverter

In [21], the use of a master inverter, responsible of injecting a high frequency rotating voltage vector (1), was proposed, the rest of inverters in the microgrid being configure to be slaves. Two high frequency signals were injected by the master inverter: the first one for islanding detection, as described in Section II; the second one to communicate the islanding condition to the rest of (slave) inverters present in the microgrid. While no interference between inverters can occur -only the master inverter injects high frequency signals-, this method has several limitations:

- Failure of the master inverter would leave the grid without the high frequency signal voltage, the rest of inverters in the grid hence losing their capability to detect islanding.

- In large microgrids, the location of the master inverter has to be carefully chosen. For inverters located far from the PCC, the variation of the high frequency impedance between grid and island condition might be small, reducing the reliability of the method. 


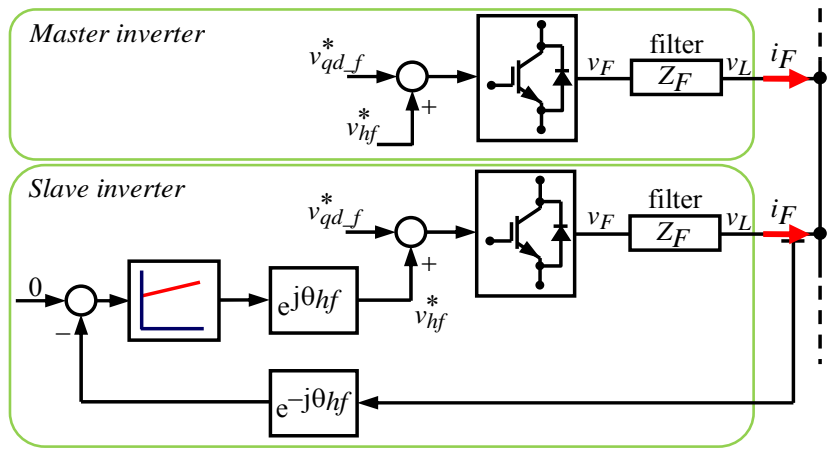

Fig. 3.- Schematic representation of the master and slave inverters implementing the infinite impedance mode. Current regulators needed for the control of the fundamental current are not shown (see Fig. 1 for details).

- Inverters including high bandwidth current regulators, e.g. active filters, might see the high frequency signal as a disturbance and partially compensate for it.

b) Dynamic (multiple) master inverter

In [22], a dynamic master assignment strategy was proposed. The signal injection by the master inverter is as in the previous case, islanding being detected from the high frequency impedance, as described in Section II. To prevent the reaction of slave inverters against the high frequency current, slave inverters include a high frequency PI current regulator (see Fig. 3). By making its command equal to zero, it is guaranteed that no high frequency current circulates through the slave inverters output, this mode of operation being named infinite impedance mode. In this mode of operation, slave inverters detect islanding/grid connected condition from the changes in their high frequency current regulator output voltage $v_{h f_{-} s}$. This also enables slave inverters detect failure of the master inverter; i.e. if $v_{h f s}{ }^{*}=0$, means that there is no master inverter injecting the high frequency voltage. This master-slave strategy has several appealing properties.

- The high frequency voltage injected by the master inverter is not corrupted by the reaction of other inverters in the grid.

- Slave inverters automatically detect failure of the master inverter. In [22], a competitive strategy for the reassignment of the master inverter role in the event of master inverter failure was defined.

- The master-inverter reassignment strategy naturally allows multiple master inverters. Slave inverters being too far from the master inverter might be unable to detect master's high frequency signal voltage. This will automatically trigger a competitive process to become master. As a result, several master inverters, distributed all over the microgrid, can operate simultaneously.

Regardless of its appealing characteristics, this strategy also has some limitations:

- The modes of operation of operation of master and slave inverters differ substantially (see Fig. 3, [21,22]), the change between both modes being triggered by the presence/absence of the high frequency voltage in the microgrid. Though the filtering to detect the presence of the high frequency voltage is simple in principle [21], this may be difficult in practice, as the signals can be corrupted by noise in the acquisition system, harmonics in the voltages due to non-linear loads, transients, etc. Spurious detection of the high frequency voltage might result in frequent, unwanted transitions between master/slave modes, of unpredictable results.

- For a given magnitude of the injected high frequency voltage at the filter output, the resulting high frequency component of the filter current $i_{F h f}(4)$, will be function of the high frequency voltage $v_{F h f}$, the output filter impedance $Z_{F}$, and the equivalent high frequency impedance $Z_{h f}$, seen at the point of connection of the inverter to the microgrid.

$i_{F h f}=\frac{v_{F h f}}{Z_{F}+Z_{h f}}$

While $v_{F h f}$, and $Z_{F}$ are known in advance, significant uncertainty exists regarding $Z_{h f}$. For inverters close to the PCC in grid operation, this impedance can be very small, the circulating high frequency current $i_{F h f}$ being therefore large, adversely impacting the power quality (THD).

To overcome aforementioned limitations, a strategy for the coordinated operation of multiple parallel connected inverters in a microgrid is proposed in the following section.

\section{IV.- Islanding detection in microgrids with multiple inverters using combined current/voltage injection}

Most of signal injection based, islanding detection methods proposed so far, inject a high frequency voltage, the island/grid condition being estimated either from the resulting high frequency current or from the high frequency impedance. However, it is also possible to inject a high frequency current, a high frequency current regulator being used for this purpose.

Current injection can be advantageous in certain cases. For inverters operating near the PCC during grid operation, the equivalent impedance will be small, what might result in a relatively large high frequency current if a constant voltage is injected. Using high frequency current injection automatically limits the resulting high frequency current. On the contrary, high frequency voltage injection would be advantageous during island operation or for inverters located far from the PCC, where injection of the rated high frequency current would require too much voltage. Combining high frequency voltage injection and high frequency current injection would therefore be beneficial for inverters experiencing large variations in the high frequency impedance seen at their terminals, as would be the case of inverters working near the PCC.

Islanding detection using combined voltage/current injection is analyzed in this section. In the proposed strategy, all the inverters synchronously inject a high frequency signal, dynamically readapting their mode of operation between voltage and current injection, depending on their location and on the grid condition. The model used for the analysis is first presented, combined voltage/current injection, synchronization of the high frequency signals, as well as the parallel operation of inverters, being analyzed in further subsections. 


\begin{tabular}{|c|c|c|c|c|c|c|c|c|c|c|c|c|c|c|c|c|c|c|c|}
\hline$v_{h f 1}$ & & $Z_{F 1}$ & 1 & 0 & 0 & 0 & 0 & 0 & 0 & 0 & 0 & $\cdots$ & 0 & 0 & 0 & 0 & 0 & 0 & $i_{F h f 1}$ \\
\hline 0 & & 1 & 0 & -1 & -1 & 0 & 0 & 0 & 0 & 0 & 0 & $\ldots$ & 0 & 0 & 0 & 0 & 1 & 0 & $v_{L h f 1}$ \\
\hline 0 & & 0 & 1 & $-Z_{L 12}$ & 0 & 0 & -1 & 0 & 0 & 0 & 0 & $\ldots$ & 0 & 0 & 0 & 0 & 0 & 0 & $i_{L h f 12}$ \\
\hline 0 & & 0 & 1 & 0 & $-Z_{l 1}$ & 0 & 0 & 0 & 0 & 0 & 0 & $\cdots$ & 0 & 0 & 0 & 0 & 0 & 0 & $i_{\operatorname{lhf} 2}$ \\
\hline$v_{h f 2}$ & & 0 & 0 & 0 & 0 & $Z_{F 2}$ & 1 & -1 & 0 & 0 & 0 & $\ldots$ & 0 & 0 & 0 & 0 & 0 & 0 & $i_{F h f 2}$ \\
\hline 0 & & 0 & 0 & 1 & 0 & 1 & 0 & -1 & -1 & 0 & 0 & $\ldots$ & 0 & 0 & 0 & 0 & 0 & 0 & $v_{L h f 2}$ \\
\hline 0 & & 0 & 0 & 0 & 0 & 0 & 1 & $-Z_{L 23}$ & 0 & 0 & -1 & $\ldots$ & 0 & 0 & 0 & 0 & 0 & 0 & $i_{L h f 23}$ \\
\hline 0 & & 0 & 0 & 0 & 0 & 0 & 1 & 0 & $-Z_{l 2}$ & 0 & 0 & $\ldots$ & 0 & 0 & 0 & 0 & 0 & 0 & $i_{l h f 3}$ \\
\hline$\cdots$ & $=$ & $\ldots$ & $\ldots$ & $\ldots$ & $\ldots$ & $\ldots$ & $\ldots$ & $\ldots$ & $\ldots$ & $\ldots$ & $\ldots$ & $\ldots$ & $\ldots$ & $\ldots$ & $\ldots$ & $\ldots$ & $\ldots$ & $\ldots$ & $\cdots$ \\
\hline$v_{h f N-1}$ & & 0 & 0 & 0 & 0 & 0 & 0 & 0 & 0 & 0 & 0 & $\cdots$ & 0 & 0 & 0 & 0 & 0 & 0 & $i_{F h f N-1}$ \\
\hline 0 & & 0 & 0 & 0 & 0 & 0 & 0 & 0 & 0 & 0 & 0 & $\cdots$ & -1 & -1 & 0 & 0 & 0 & 0 & $v_{L h f N-1}$ \\
\hline 0 & & 0 & 0 & 0 & 0 & 0 & 0 & 0 & 0 & 0 & 0 & $\ldots$ & $-Z_{L N-1 N}$ & 0 & 0 & -1 & 0 & 0 & $i_{L h f N-1 N}$ \\
\hline 0 & & 0 & 0 & 0 & 0 & 0 & 0 & 0 & 0 & 0 & 0 & $\ldots$ & 0 & $-Z_{L N}$ & 0 & 0 & 0 & 0 & $i_{l h f N-1}$ \\
\hline$v_{h f N}$ & & 0 & 0 & 0 & 0 & 0 & 0 & 0 & 0 & 0 & 0 & $\cdots$ & 0 & 0 & $Z_{F N}$ & 1 & 0 & 0 & $i_{l h f N}$ \\
\hline 0 & & 0 & 0 & 0 & 0 & 0 & 0 & 0 & 0 & 1 & 0 & $\cdots$ & 1 & 0 & 1 & 0 & -1 & -1 & $v_{L h f N}$ \\
\hline 0 & & 0 & -1 & 0 & 0 & 0 & 0 & 0 & 0 & 0 & 0 & $\cdots$ & 0 & 0 & 0 & 1 & $-Z_{L N 1}$ & 0 & $i_{L h f N 1}$ \\
\hline 0 & & 0 & 0 & 0 & 0 & 0 & 0 & 0 & 0 & 0 & 0 & $\cdots$ & 0 & 0 & 0 & 1 & 0 & $-Z_{I N}$ & $i_{l h f N}$ \\
\hline
\end{tabular}

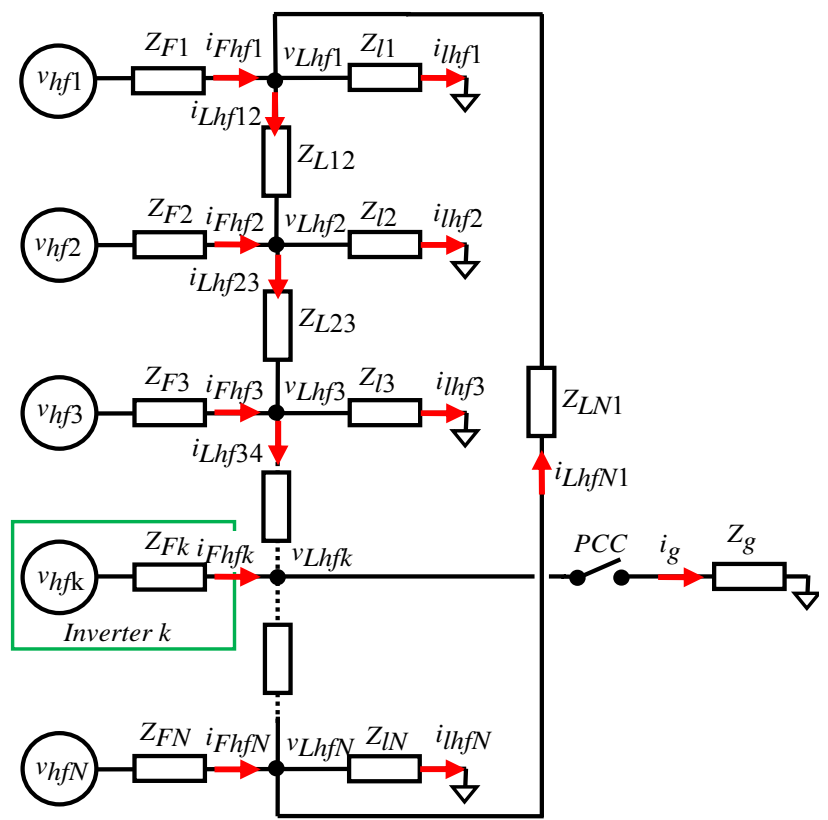

Fig. 4.- Generic microgrid architecture.

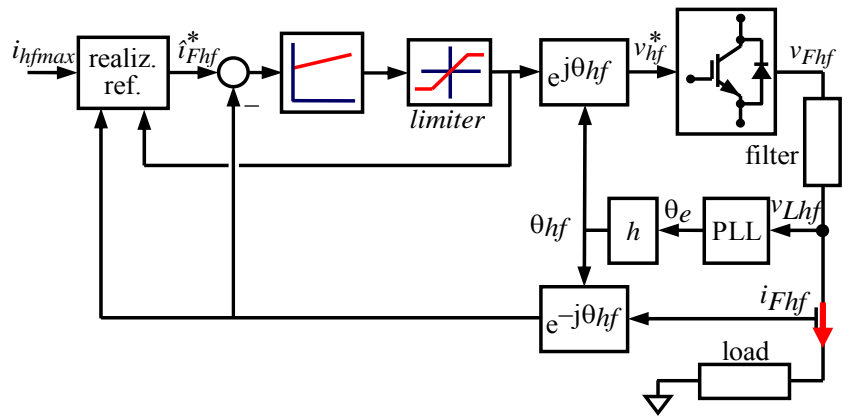

Fig. 5.- Power converter, including high frequency current regulator, realizable references block, and PLL for the synchronization of the high frequency signal with the grid fundamental voltage.

\section{a) Grid model}

The microgrid model shown in Fig. 4 will be used for analysis and simulation purposes. It is observed that the microgrid has a radial configuration, with node $k$ being the point of common coupling (PCC) [1]. The mathematical model of the grid is given by (5), where $Z_{F k}$ is the filter impedance between each inverter participating in the strategy (i.e. injecting a high frequency voltage/current) and the microgrid, $Z_{L k-1 k}$ is the line impedance between nodes $k-1$ and $k, Z_{l k}$ is the load impedance in node $k, Z_{g}$ is the grid impedance and $v h f k$ is the high frequency voltage injected by the inverter at node $k$.

The model in Fig. 4 and (5) can be used to analyze the system response to the high frequency signals by just replacing the impedances and voltage sources by their corresponding high frequency values. It is noted that the grid voltage as well as the voltage for any generating unit or load not injecting the high frequency voltage/current, can be made equal to zero, as it does not contain a high frequency component. Therefore, the grid as well as passive loads or generators which do not inject a high frequency signal, are replaced by an equivalent impedance connected to ground. As consequence of this, even in the event that no net power flows through the PCC in grid operation (the microgrid voltage at the PCC in island condition exactly matches the grid voltage), the grid impedance $Z_{g}$ is connected to ground in the grid side, being therefore measurable (see fig. 4). It is also noted that the model presented in Fig. 4 assumes that there is a load connected at each node, which corresponds to the microgrid case discussed in Section I.

The model in Fig. 3and (5) can be easily adapted to the multi-inverter case discussed in section I, where most of all of the power is evacuated to the grid at the PCC (e.g. distributed photovoltaic generation systems [24]), by making the load impedances equal to zero $\left(Z_{l_{X}}=0\right)$. 


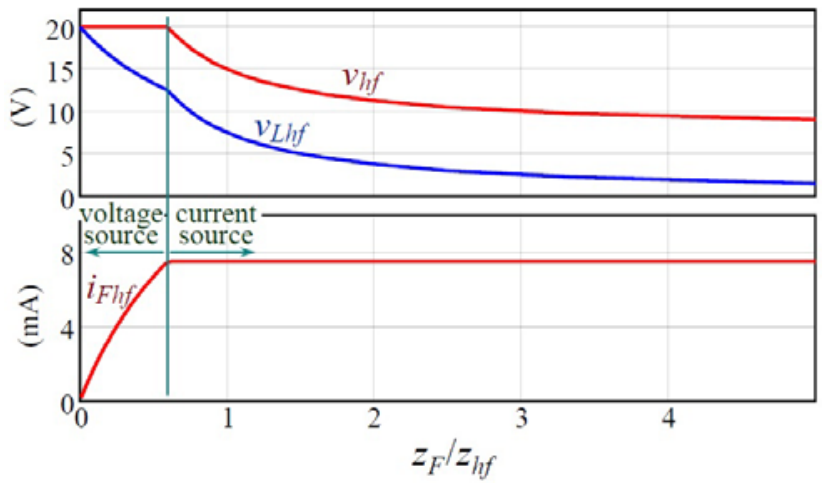

Fig. 6.- Combined Voltage source and Current source modes of operation.

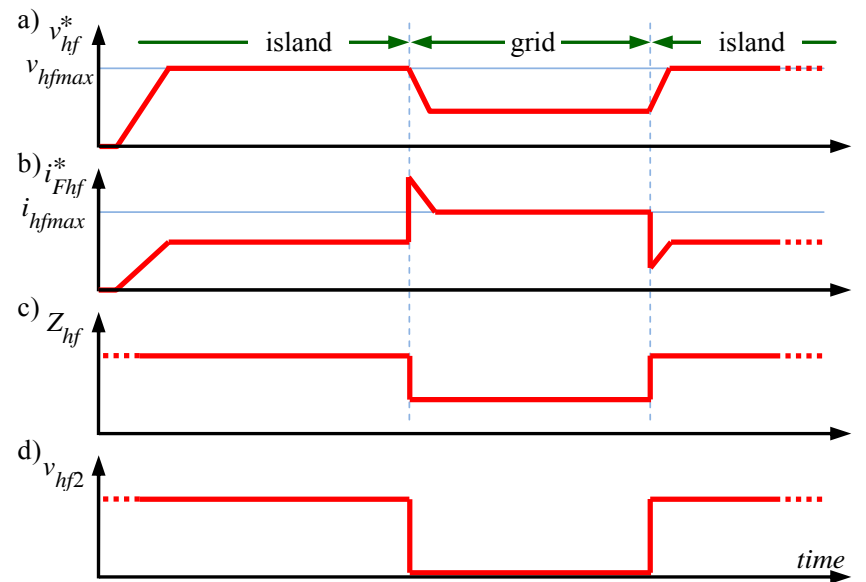

Fig. 7.- Schematic representation of the transition between island and grid conditions. a) magnitude of the high frequency voltage and b) current injected for islanding detection; c) high frequency impedance; d) secondary high frequency voltage.

It is finally noted that (5) assumes that the inverters operate as a Voltage source. For inverters operating in the Current source mode, (5) can be algebraically rearranged by moving the corresponding current $i_{F k}$ to the left term (inputs vector) and the inverter voltage $v h f k$ to the right side (outputs vector) of the equation respectively.

b) Combined high frequency Voltage/Current signal injection

A means to combine current and voltage injection providing automatic, smooth transition between both modes of operation, is to add a saturation mechanism as a part of the high frequency current regulator [25]. This mechanism, named realizable references, is schematically shown in Fig. 5. A PI current regulator implemented in a reference frame synchronous with the high frequency signal is used, the current command being $\hat{l}_{F h f}^{*}$ (see Fig. 5). If the voltage $v h f$ required to supply the commanded current is lower than the established voltage limit (limiter in Fig. 5), the current injection mode works normally. This will occur if $Z_{h f}$ is small. On the contrary, if the voltage $v h f$ goes beyond the established voltage limit $v$ hfmax, the voltage effectively injected is limited to this value. This will be the case when $Z_{h f}$, is large. If this occurs, the current command is recalculated to make it consistent with the high frequency voltage commanded to the inverter $v$ hfmax.
In each sampling period, the regulator equation is computed digitally in the regular way (6) to (8), (Euler's approach was used in (8)), assuming that the actual current command is realizable, i.e. will not saturate the output voltage.

$$
\begin{aligned}
& \hat{l}_{F h f(k)}^{*}=i_{h f m a x} \\
& e_{F h f(k)}=\hat{\imath}_{F h f(k)}^{*}-i_{F h f(k)} \\
& v_{h f(k)}^{*}=v_{h f(k-1)}^{*}+\left(K_{p}+K_{i} T\right) e_{F h f(k)}-K_{p} e_{F h f(k-1)}
\end{aligned}
$$

If the obtained voltage does not need to be limited, no further action is required. On the contrary, if the output voltage has to be limited (9), a new, realizable reference, is computed by using the regulator equations backwards, (10) and (11). The equations are written trying to clarify the technique rather than with an optimal implementation.

$$
\begin{aligned}
& \text { If } v_{h f(k)}^{e^{*}}>v h f \text { max } \rightarrow v_{h f(k)}^{e^{*}}=v h f \text { max } \\
& e_{h f(k)}^{e}=\frac{v_{h f(k)}^{e^{*}}-v_{h f(k-1)}^{e^{*}}+K_{p} e_{h f(k-1)}^{e}}{K_{p}+K_{i} T} \\
& \hat{\imath}_{h f(k)}^{e^{*}}=e_{h f(k)}^{e}+i_{h f(k)}^{e}
\end{aligned}
$$

After computing equations (10) and (11), the current error and the output voltage will be consistent. The realizable reference reflects therefore a current command consistent with the controller voltage limit. Because of its design basis, the realizable reference does not depend on any load parameter.

Fig. 6 schematically shows the injected high frequency voltage at the inverter terminals (commanded), the high frequency voltage at the filter output, and the resulting high frequency current, as a function of the relationship between the filter impedance $Z_{F}$ and the equivalent high frequency impedance $Z_{h f}$. When the impedance $Z_{h f}$ is large, the inverter output voltage is limited, i.e. operates as a Voltage source. As $Z_{h f}$ decreases $\left(Z_{F} / Z_{h f}\right.$ increases), once the high frequency current reaches its rated value, the inverter works in the current control mode, behaving therefore as a Current source.

For the implementation of the strategy, ihfmax is selected first. The relationship between ihfmax and vhfmax is set to be (12), where $Z_{F}$ is the (known) filter impedance, and $\hat{Z}_{h f}$ is the preset value of the high frequency impedance at which the change between Voltage source and Current source occurs. For the simulation and experiments carried out in this paper, $i_{h f m a x}$ was set to $1.5 \%$ of the rated current and $\hat{Z}_{h f}$ to $0.35 \cdot Z_{F}$, vhfmax for the limiter block in Fig. 5 being obtained from (12).

$$
v_{h f \max }=i_{h f \max }\left(Z_{F}+\hat{Z}_{h f}\right)
$$

Fig. 7 shows the simulated response of the method. During island mode, the voltage is limited (Fig. 7a), the high frequency current command being recalculated using (9)-(11) to be consistent with the voltage. During grid operation, due to the reduction of the impedance seen by the inverter, current injection works normally, the high frequency current command being equal to the nominal value ihfmax. 
Grid-connected mode

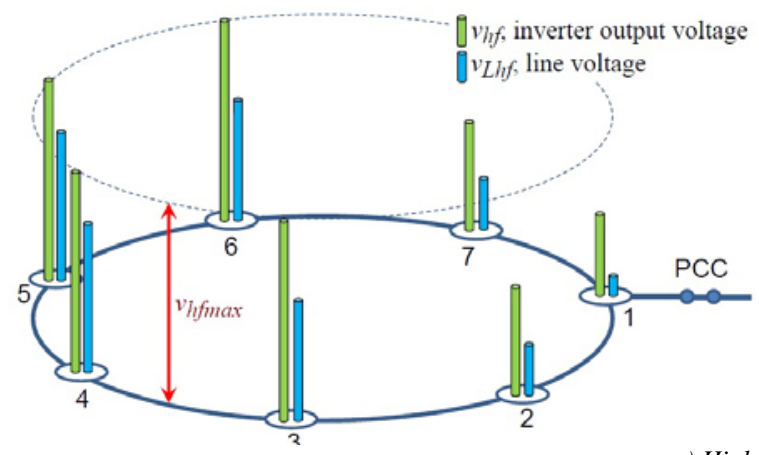

Islanding mode

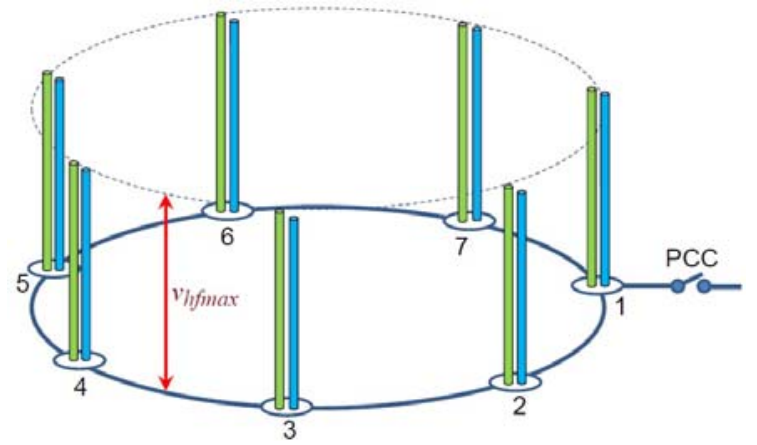

a) High frequency voltage
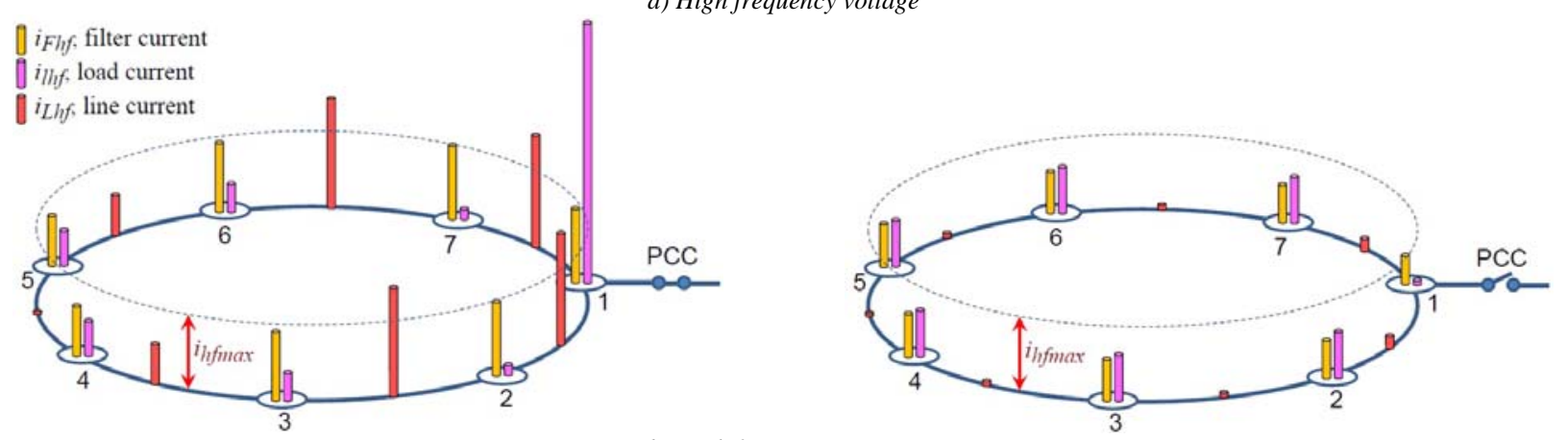

b) High frequency current
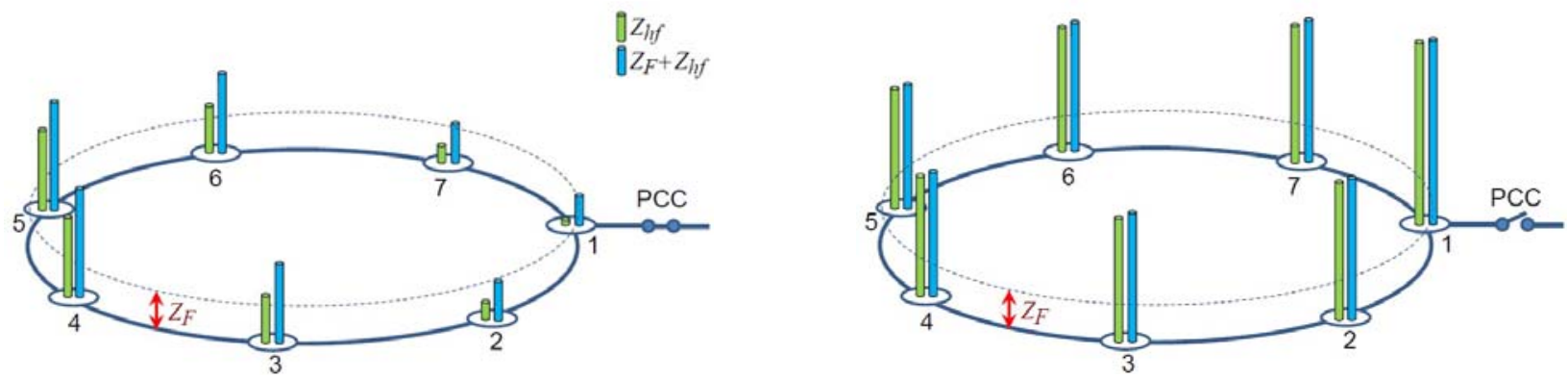

c) High frequency impedance

Fig. 8.- Schematic representation of the microgrid, operating in grid-connected (left) and islanding (right) modes. a) high frequency voltages, b) high frequency currents and c) high frequency impedances. The load current in node \#1 corresponds to the high frequency current circulating into the grid. The bars in subplots a) to b) are referred to the maximum high frequency voltage $v_{h f m a x}$, current $i_{h f m a x}$ and output filter impedance $Z_{F}$, respectively.

It is remarked that the transition between Voltage and Current source modes occurs smoothly, no reconfiguration of the high frequency signal injection mechanism being needed (Fig. 7c). Experimental results showing the operation of this method are provided in Section $\mathrm{V}$.

\section{c) Synchronization of the high frequency signal injection}

If multiple inverters inject simultaneously, synchronization of the high frequency signal for each inverters is highly advisable, as this will reduce the differential high frequency voltage among inverters. Otherwise, the resulting differential high frequency voltage among inverters will result in large, time varying, high frequency currents, adversely affecting to the power quality (THD), also making more challenging the detection of grid/island condition. Phase-synchronization of the inverters can be done easily by selecting the high frequency voltage to be an integer multiple of the grid frequency. A cascade complex coefficient filter with complex PLL (CCCFCPLL) [26] was used for this purpose (see Fig. 5).

\section{d) Parallel operation of the inverters}

Inverters using the combined Voltage source/Current source modes can be directly connected in parallel. The result is a microgrid in which all the inverters participating in the islanding detection strategy, inject the high frequency signal, each inverter automatically selecting the mode of operation, depending on the high frequency impedance $Z_{h f}$ present at its terminals. Fig. 8 show an example obtained using the model in Fig. 4, (5), for the case of grid (left) and island operation (right). In the example shown in Fig. 8, the microgrid consists of seven nodes, radially connected, the PCC being in node \#1. Each inverter uses the configuration shown in Fig. 5. The system parameters are the same as for the experimental results (see Table I). The bars in Fig. 8a show the inverter 


\begin{tabular}{|l|l|}
\hline \multicolumn{2}{|c|}{ Table I. } \\
\hline Grid & $380 \mathrm{~V}, 50 \mathrm{~Hz}, \mathrm{~S}_{\mathrm{cc}}=2 \mathrm{MVA}$ \\
\hline Inverters & $380 \mathrm{~V}, 30 \mathrm{kVA}, 10 \mathrm{kHz}$. \\
\hline Load & $15 \mathrm{~kW}$ \\
\hline${ }_{\text {h } f \text { max }}$ & $13 \mathrm{~V}(0.0325 \mathrm{pu}), 250 \mathrm{~Hz}\left(5 \times \omega_{e}\right)$ \\
\hline ih $_{\text {fmax }}$ & $1 \mathrm{~A}(0.0155 \mathrm{pu})$ \\
\hline Line impedance $Z_{L}$ & $2 \Omega$ \\
\hline LCL filter & $2.4 / 2.3 \mathrm{mH}$ (inverter/grid side), C=30uF \\
\hline
\end{tabular}

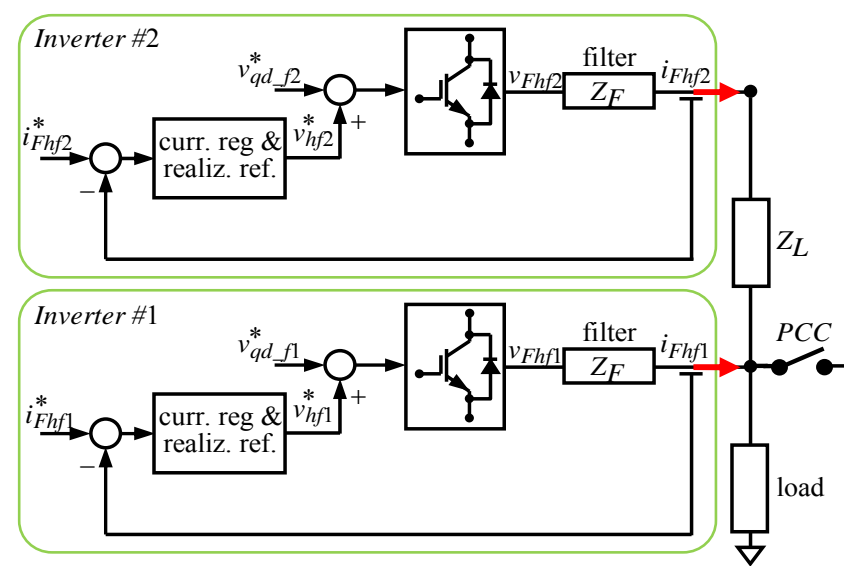

Fig. 9.- Experimental setup, consisting of two inverters. Current regulators needed for the control of the fundamental current are not shown.

output voltage $v_{h f}$ and line voltage $v_{L h f}$ (filter output voltage) for each node. Fig. $8 \mathrm{~b}$ shows the filter, line and load high frequency current. The limits for the high frequency voltage, vhfmax, and current, ihfmax, (see Fig. 6 and 7), are indicated in Fig. $8 \mathrm{a}$ and $8 \mathrm{~b}$. It is noted that node \#1 corresponds to the PCC, the load current in this node coinciding therefore with the high frequency current circulating into the grid.

It is observed from Fig. 8 a-left and b-left that in gridconnected mode, inverters in nodes \#1, \#2, and \#7 operate in the Current source mode. Due to their closeness to the PCC, the impedance seen by these inverters is small, their high frequency current being limited to ihfmax. On the contrary, nodes \#3 to \#6 operate in the Voltage source mode, as they are further from the PCC. It is remarked again that the transition between Voltage source and Current source occurs smoothly, no reconfiguration of the injection mechanism being needed.

\section{e) Islanding detection and islanding notification}

Islanding is detected from the measured high frequency impedance. Fig. 8c shows the high frequency impedance seen at the inverters terminal (13) and filters output (14).

$$
\begin{aligned}
& Z_{F}+Z_{h f}=\frac{v_{h f}}{i_{F h f}} \approx \frac{v_{h f}^{*}}{i_{F h f}} \\
& Z_{h f}=\frac{v_{L h f}}{i_{F h f}}
\end{aligned}
$$

Since $Z_{h f}$ is the variable reflecting the changes between grid and islanding modes, (14) would be used in principle for islanding detection. However, using (13) has the advantage that the commanded high frequency voltage can be used, instead of the high frequency component of the measured line voltage needed in (14), slightly simplifying the required signal processing. Still both options are expected to give similar results.

Some expected facts are observed in Fig. 8. For the case of grid-connected operation, the high frequency impedances for each node (both $Z_{h f}$ and $Z_{F}+Z_{h f}$ in Fig. 8c) depend on the node location, relative to the PCC, the impedance for nodes closer to the PCC being significantly smaller. Comparing the nodes impedances for the case of grid (Fig. 8c-left) and island (Fig. 8c-right), the nodes closer to the PCC experience the largest variations of the high frequency impedance between grid and island connection.

Currently, islanding detection is based on the rate of variation of $Z_{h f}$ with time, i.e. $\Delta Z_{h f} / \Delta t$ [4-13]. Comparison of $Z_{h f}$ with a threshold might also potentially be used [21]. Islanding detection for nodes closer to the PCC will be easier and more reliable. On the contrary, islanding detection might be difficult for nodes far from the PCC in large microgrids. Thus, inverters working in the Current source mode would be in principle better candidates to detect islanding than inverters working in the Voltage source mode.

A possible strategy would be to enable only inverters working in the Current source to inform of the grid condition to the rest of inverters present in the microgrid, using a secondary high frequency signal for this purpose. Inverters working in the Current source mode would detect islanding by measuring a positive $\Delta Z_{h f}$ over a predefined time window. Once islanding is detected, they would inject the secondary high frequency signal $v h f 2$. On the contrary, if inverters injecting the secondary high frequency voltage detect the change between islanding and grid-mode from the measured $\Delta Z_{h f}$, they would discontinue injecting the secondary high frequency voltage. Injection of the secondary high frequency voltage is schematically shown in Fig. $7 d$, details on the use of the method can be found in [21,22].

This strategy has not been studied in detail, further analysis would be needed to verify its viability and performance.

\section{V.- Experimental results}

The proposed islanding detection strategy has been tested using the experimental setup shown in Fig. 9, the parameters being summarized in Table I. Both inverters are identical, they use IGBTs, all the control (current regulators, synchronization, etc.) being implemented on a TMS320F28335 DSP.

Fig. 10 shows the transition between island and grid modes. Fig. 10a and 10c show the commanded and filter output voltages for both inverters, while Fig. 10b and 10d show the corresponding high frequency currents. The magnitude of the high frequency current ihfmax is set to $1 \mathrm{~A}$ (1.5\% rated current). It is observed from Fig. 10b that during islanding, both inverters operate in the Voltage source mode, with $v_{\text {hfmax }}=13 \mathrm{~V}$. During island operation, Inverter \#1 changes its mode of operation to the Current source mode, its high frequency current being limited to ihfmax, while Inverter \#2 keeps operating in the Voltage source mode. This is due to the line impedance between Inverter \#2 and the PCC. 

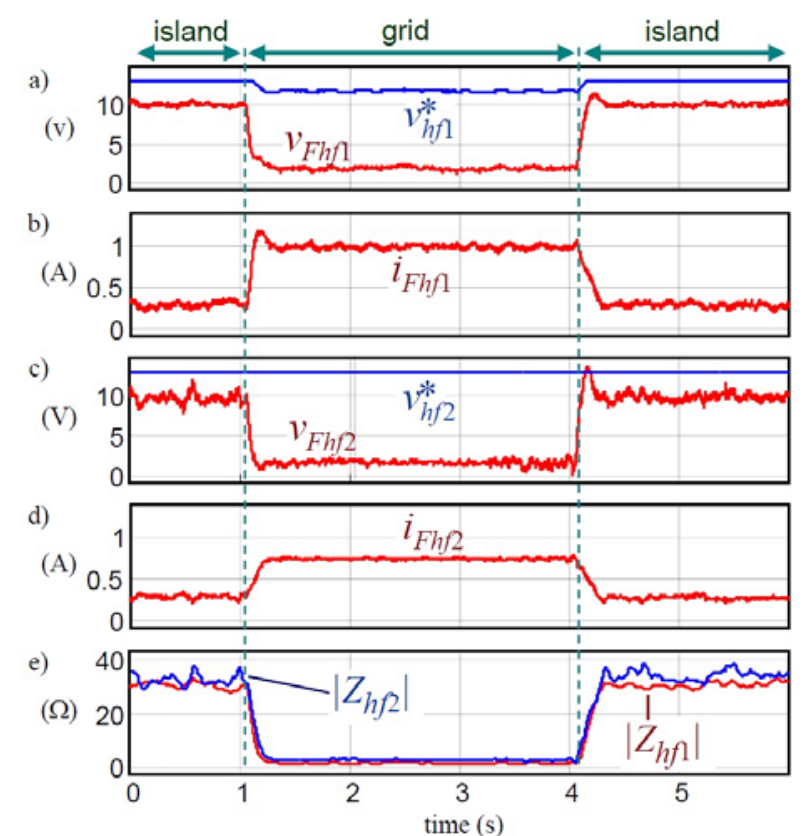

Fig. 10.- Experimental results showing the response of the proposed method during island-grid transitions.

Fig. 10e and 10f show the magnitude of the corresponding high frequency impedances. It is observed that for this particular case, the change between grid and island modes is readily detected by both inverters. This is due to the fact that the line impedance $Z_{L}$ is small compared to the impact that the change between grid and island operation has on the equivalent impedance $Z_{h f}$. It is noted however that, for the case of large microgrids, inverters far from the PCC might experience significantly smaller variations of the equivalent impedance, making it less reliable for islanding detection purposes.

\section{VI.- Conclusions}

A method to detect islanding in grids with multiple parallelconnected inverters has been presented in this paper. In the proposed method, all the inverters participating in the strategy inject the high frequency signal. The inverters can operate in two different modes of operation: Current injection mode and Voltage injection. Combined used of modes has two advantages: the adverse impact that the injection of the high frequency signal has on the power quality (THD) is limited, also it provides a mean to evaluate the suitability of a particular inverter to detect islanding, what can be important in large microgrids. An important feature of the proposed method is that transition between Current injection and Voltage injection modes of operation occurs automatically and smoothly, no change in the configuration of the inverters being required. Simulation and experimental results have been presented to demonstrate the viability of the proposed method.

\section{References}

[1] Guerrero, J.M.; Chandorkar, M.; 3, T.; Loh, P.C. " Advanced Control Architectures for Intelligent Microgrids-Part I: Decentralized and Hierarchical Control," IEEE Trans. on Ind. Electr., vol.60, no.4, pp. 1254,1262, April 2013
[2] Massoud, A.M.; Ahmed, K.H.; Finney, S.J.; Williams, B.W., "Harmonic distortion-based island detection technique for inverterbased distributed generation," IET Renewable Power Generation, vol.3, no.4, pp.493,507, December 2009

[3] J.M. Lee, Islanding Detection Methods for Microgrids, Ms. Thesis, University of Wisconsin-Madison, 2011

[4] IEEE, "IEEE Standard for Interconnecting distributed resources with electric power systems," IEEE Std. 1547, 2003.

[5] IEC, "IEC Photovoltaic (PV) systems. Characteristics of the utility interface," IEC Std. 61 727, 2004.

[6] ENEL, "DK 5940 Criteria for connection of generation systems to ENEL low voltage distribution networks," 2006.

[7] IEEE Std. 929-2000, IEEE Recommended Practice for Utility Interface of Photovoltaic (PV) Systems, IEEE Standards Coordinating Committee 21 on Photovoltaics, New York, NY, Apr. 2000.

[8] UL1741, UL Standard for Safety for Static Converters and Charge Controllers for Use in Photovoltaic Power Systems, Underwriters Laboratories, May 7, 1999, revised June 2001.

[9] IEC 62116, Testing Procedure of Islanding Prevention Measures for Grid Connected Photovoltaic Power Generation Systems, International Electrotechnical Commission.

[10] DIN-VDE, "Automatic Disconnection Device Between a Generator and the Low-Voltage Grid," DIN-VDE Std. 0126-1-1, 2005.

[11] G77 - Recommendations for the Connection of Inverter- Connected Single-Phase Photovoltaic (PV) Generators up to 5kVA to Public Distribution Networks.

[12] ÖVE/Önorm E 2750 "Photovoltaische Energieerzeugungsanlagen Sicherheitsanforderungen ("Photovoltaic power generating systems safety requirements").

[13] VSE Sonderdruck Abschnitt 12 'Werkvorschriften über die Erstellung von elektr. Installation' Elektrische Energieerzeugungsanlagen Completes VSE 2.8d-95.

[14] A. Timbus, A. Oudalov, C. N.M. Ho, "Islanding Detection in Smart Grids" IEEE-ECCE'10, pp.3631 - 3637, Sep. 2010.

[15] R. Teodorescu, M. Liserre, P. Rodriguez and F. Blaabjerg, Grid Converters for Photovoltaic and Wind Power Systems, Wiley-IEEE 2011.

[16] M. Ciobotaru, R. Teodorescu, P. Rodriguez, A. Timbus and F. Blaabjerg, "On-line Grid Impedance Estimation for Single Phase GridConnected Systems Using PQ Variations", IEEE-PESC, pp.2306-2312, June 2007.

[17] A. V. Timbus, R. Teodorescu and U. Borup, "Online Grid Impedance Measurement Suitable for Multiple PV Inverters Running in Parallel”, IEEE-APEC'06, pp.907-911, March 2006.

[18] L. Asiminoaei, R. Teodorescu, F. Blaabjerg and U. Borup, "A Digital Controlled PV-Inverter with Grid Impedance Estimation for ENS Detection”, IEEE Trans. on Ind. Appl., 20(4):1480-1490, Nov.-Dec. 2005.

[19] L. Asiminoaei, R. Teodorescu, F. Blaabjerg and U. Borup, "A New Method of On-Line Grid Impedance Estimation for PV Inverter", IEEE-APEC'04, pp.1527-1533, Sept. 2004

[20] M. Ciobotaru, R. Teodorescu and F. Blaabjerg, "On-line Grid Impedance Estimation Based on Harmonic Injection for GridConnected PV Inverter", IEEE-ISIE, pp.2473-2442, June 2007.

[21] Reigosa, D.D.; Briz, F.; Charro, C.B.; Garcia, P.; Guerrero, J.M., "Active Islanding Detection Using High-Frequency Signal Injection, IEEE Trans. on Ind. Appl, vol.48, no.5, pp.1588,1597, Sept.-Oct. 2012

[22] Reigosa, D.; Briz, F.; Guerrero, J.; Garcia, P.; Blanco Charro, C., "Active Islanding Detection for Multiple Parallel-Connected InverterBased Distributed Generators Using High Frequency Signal Injection, IEEE Trans. on Power Electronics, pending of publication.

[23] Hernandez-Gonzalez, G.; Iravani, R., "Current injection for active islanding detection of electronically-interfaced distributed resources," IEEE Trans. on Power Delivery, vol.21, no.3, pp.1698-1705, July 2006

[24] Yan Zhou; Hui Li; Liming Liu, "Integrated Autonomous Voltage Regulation and Islanding Detection for High Penetration PV Applications", IEEE Trans. on Power Electr., vol.28, no.6, pp.2826,2841, June 2013

[25] Briz, F.; Diez, A.; Degner, M.W.; Lorenz, R.D., "Current and flux regulation in field-weakening operation, IEEE Trans. on Ind. Appl., vol.37, no.1, pp.42,50, Jan/Feb 2001

[26] C. Blanco, D. Reigosa, F. Briz, J. M. Guerrero, y P. Garcia, "Grid synchronization of three-phase converters using cascaded complex vector filter PLL", in 2012 IEEE Energy Conversion Congress and Exposition (ECCE), pp. 196-203, Sept. 2012. 